\title{
Tarifpolitischer Jahresbericht 2012: Höhere Tarifabschlüsse und Erfolge bei Leiharbeit und Ausbildung
}

\begin{abstract}
Die Tarifrunde 2012 führte lohnpolitisch zu positiven Ergebnissen. Wie schon im Vorjahr gelang es den Gewerkschaften erneut, höhere Tarifabschlüsse durchzusetzen. Angesichts der verhaltenen Entwicklung der Verbraucherpreise resultierte daraus ein realer Anstieg der Tarifverdienste um 0,7 \%. Auch bei qualitativen Tarifthemen gab es Fortschritte: Der Lohnabstand der Leiharbeitsbeschäftigten zu den Stammbeschäftigten konnte in einigen Branchen verringert und die Bedingungen für die Übernahme der Ausgebildeten konnten verbessert werden. Ob der Lohntrend sich 2013 fortsetzt, ist offen, denn die aktuelle Tarifrunde steht unter unsicheren wirtschaftlichen Vorzeichen.
\end{abstract}

REINHARD BISPINCK, WSI-TARIFARCHIV

\section{Das Tarifjahr im Überblick}

\subsection{Rahmenbedingungen und Tarifforderungen}

Die vergleichsweise positive tarifpolitische Entwicklung im Jahr 2012 war nicht von vornherein absehbar, denn die ökonomischen Rahmenbedingungen der Tarifrunde gestalteten sich nicht günstig. Insofern ähnelte der Beginn der Tarifrunde 2012 der Situation des Vorjahres. Im Jahr 2011 verlangsamte sich das zunächst sehr kräftige Wachstum deutlich, im vierten Quartal war sogar ein Schrumpfen des Bruttoinlandsprodukts (BIP) um 0,1 \% zu verzeichnen, und die Perspektiven für 2012 sahen nach Ansicht der Wirtschaftsforschungsinstitute keinesfalls rosig aus. Mehrheitlich schraubten sie ihre Wachstumsprognosen auf 0,5-1,0\% zurück, zum Teil wurde ein Rückgang des BIP für 2012 angenommen (IMK 2011). Dementsprechend abwartend gingen die Gewerkschaften zunächst an die Vorbereitung und Aufstellung der konkreten Tarifforderungen heran. Zu den positiven Einflussfaktoren zählte hingegen die Entwicklung auf dem Arbeitsmarkt. Die Arbeitslosenquote ging im Jahresverlauf 2011 von 7,9 auf 6,6 \% zurück, die saisonbereinigte Zahl der Arbeitslosen sank von 3,1 auf 2,9 Mio., die Zahl der Erwerbstätigen stieg im Jahresdurchschnitt um 1,0\%, die sozialversicherungspflichtige Beschäftigung um 1,9\% (BA 2013). Ähnlich wie bereits 2011 gab es auch zu Beginn der Tarifrunde 2012 deutliche Unterstützung für die Forderung nach kräftigen Lohnsteigerungen vonseiten der Politik, aber auch aus dem Bereich der Wirtschaftsforschungsinstitute. Eine wichtige Rolle spielte dabei die „Gerechtigkeitsdebatte“, die sich an den exorbitanten Steigerungen der Managerverdienste insbesondere bei den DAX-Konzernen entzündete (Spiegel Online 2012).

Zusätzlich zu den Lohn- und Gehaltsforderungen stellten die Gewerkschaften in einigen Branchen auch weitere qualitative Forderungen auf, die auf die Begrenzung prekärer Arbeit und die Durchsetzung von Bedingungen „Guter Arbeit“ zielten. Geprägt wurde die Tarifrunde 2012 maßgeblich von den Tarifbewegungen im öffentlichen Dienst (Bund und Gemeinden), in der Metall- und Elektroindustrie und in der chemischen Industrie. Das Konfliktniveau war relativ hoch, wie die umfangreichen Warnstreiks im öffentlichen Dienst und in der Metallindustrie belegen.

Die Lohn- und Gehaltsforderungen der Gewerkschaften bewegten sich in der Tarifrunde 2012 überwiegend zwischen 6 und $7 \%$ und damit in etwa wie im Jahr 2011 (Überischt 1). Die Gewerkschaft Nahrung, Genuss, Gaststätten (NGG) forderte für ihre Branchen zwischen 5 und $6 \%$ sowie tarifliche Mindestentgelte von 8,50€/Std. $6 \%$ forderten die Gewerkschaften auch in den Branchen chemische Industrie, Bankgewerbe sowie in der Landwirtschaft (hier: 5,9 \%). 6,5\% betrug die Forderung in der Metall- und Elektroindustrie sowie bei Volkswagen und bei der Deutschen Telekom AG. Im öffentlichen Dienst (Bund und Gemeinden) forderte ver. di ebenfalls $6,5 \%$, jedoch mindestens $200 €$. Das be- 
deutete für die unteren Entgeltgruppen Erhöhungen um bis zu $10 \%$ und mehr. 7 \% Tariferhöhung forderte ver.di bei der Deutschen Post AG und im privaten Verkehrsgewerbe Baden-Württemberg. Erkennbar geringer fiel mit $5 \%$ die Tarifforderung für die Textil- und Bekleidungsindustrie aus.

Der zeitliche Ablauf der Tarifrunde ergab sich aus folgenden Kündigungsterminen für die Lohn- und Gehaltstarifverträge:

- Ende Dezember 2011 liefen die Tarifverträge für die Deutsche Post AG und bei den Ortskrankenkassen aus, Ende Januar 2012 folgte die Deutsche Telekom AG;

- im Februar 2012 endete die Laufzeit der Verträge im öffentlichen Dienst (Bund und Gemeinden) sowie im Bankgewerbe;

- Ende März folgten die Metall- und Elektroindustrie sowie einige Bereiche des privaten Verkehrsgewerbes;

- Ende April standen Verträge in verschiedenen Regionen des Kfz-Gewerbes sowie des Hotel- und Gaststättengewerbes zur Verhandlung an;

- Ende Mai folgte die Volkswagen AG; ebenfalls Ende Mai sowie Ende Juni liefen die Verträge in der chemischen Industrie aus;

- Ende August liefen die Verträge in der Papier und Pappe verarbeitenden Industrie aus und im September folgten Bereiche des privaten Verkehrsgewerbes, der Kunststoffverarbeitung und der Energieversorgung, im Oktober die Textil- und Bekleidungsindustrie sowie die Landwirtschaft.

\subsection{Abschlüsse}

Wegen der lang laufenden Abschlüsse aus den Vorjahren gab es in zahlreichen Branchen im Jahr 2012 keine Lohnrunde. Dazu zählen u. a. der Einzel- und Großhandel, das Bauhauptgewerbe, die Druckindustrie, das Versicherungsgewerbe sowie der öffentliche Dienst im Bereich der Länder. Ein Blick auf ausgewählte Tarifabschlüsse zeigt folgendes Bild (Übersicht 1):

März: Den ersten großen Abschluss legte der öffentliche Dienst, Bund und Gemeinden am 31.3. vor. Er kam nach umfangreichen bundesweiten Warnstreiks zustande und sieht eine Tarifanhebung von 3,5 \% ab 1.3.2012 vor sowie zwei Stufenanhebungen von jeweils 1,4\% ab 1.1.2013 und 1.8.2013. Die Gesamtlaufzeit beträgt 24 Monate.

Mai: In der Metall- und Elektroindustrie vereinbarte die IG Metall nach umfangreichen Warnstreiks mit dem Pilotabschluss in Baden-Württemberg am 19.5.2012 nach einem Nullmonat eine Tarifanhebung von 4,3 \% bei einer Laufzeit von insgesamt 13 Monaten. Der Tarifabschluss bei Volkswagen vom 31.5.2012 sah ein ähnliches Volumen (ohne Nullmonat) vor.

In der chemischen Industrie sieht der Abschluss vom 24.5.2012 nach einem Nullmonat eine tarifliche Entgeltsteigerung von $4,5 \%$ für 18 Monate vor.
Juni: Für das Bankgewerbe konnte ver.di eine Tarifanhebung von 2,9 \% ab 1.7.2012, eine Stufenerhöhung von 2,5\% ab 1.7.2013 sowie eine Pauschalzahlung von $350 €$ für März bis Juni erreichen. Die Gesamtlaufzeit beträgt 26 Monate.

September: Für die Papier und Kunststoff verarbeitende Industrie schloss ver.di ein Tarifabkommen ab, das nach zwei Nullmonaten eine Tarifanhebung um 3,1\%ab 1.11.2012 und eine weitere Steigerung von 3,0 \% ab 1.11.2013 bei einer Laufzeit von 24 Monaten vorsieht.

November: In der Textil- und Bekleidungsindustrie vereinbarten die Tarifparteien nach zwei Nullmonaten eine Pauschale von $240 €$ für vier Monate sowie eine Tarifanhebung von 3,0 \% ab 1.5.2013 und weitere 2,0 \% ab 1.6.2014 bei einer Gesamtlaufzeit von 24 Monaten.

Was die qualitativen Tarifforderungen betrifft, sind folgende Ergebnisse hervorzuheben:

- In der Metallindustrie wurde die unbefristete Übernahme der Ausgebildeten als Grundsatz festgeschrieben und die Mitspracherechte des Betriebsrates bei Leiharbeit wurden verbessert. Außerdem einigte sich die IG Metall mit den beiden Zeitarbeitsverbänden BAP und iGZ auf tarifliche Branchenzuschläge von 15 bis $50 \%$, gestaffelt nach ununterbrochener Beschäftigungsdauer im Kundenbetrieb. Vergleichbare Regelungen wurden auch in anderen Branchen abgeschlossen.

- In der chemischen Industrie stand die Weiterentwicklung des Tarifvertrages „Lebensarbeitszeit und Demografie“ auf der Tagesordnung. Vereinbart wurden eine Aufstockung der betrieblichen Demografiefonds sowie Regelungen zur Arbeitszeitgestaltung, insbesondere für ältere Beschäftigte. - Im Bankgewerbe vereinbarte ver.di mit dem Arbeitgeberverband in Anknüpfung an frühere Regelungen eine Erklärung zum Gesundheitsschutz der Beschäftigten, die auch ein jährliches Monitoring vorsieht (siehe auch Roach 2012). - Im Bereich der tariflichen Mindestlöhne nach dem Entsendegesetz und dem Arbeitnehmerüberlassungsgesetz konnten in neun von elf Branchen Anhebungen durchgesetzt werden, die sich zwischen 1,0 und 14,9\% bewegen.

\section{Statistik der tariflichen Lohn- entwicklung}

Die DGB-Gewerkschaften schlossen 2012 in ganz Deutschland Lohn- und Gehaltstarifverträge für rund 8,9 Mio. Beschäftigte ab, davon für rund 7,9 Mio. in den alten und 1,1 Mio. in den neuen Bundesländern. Das entspricht rund knapp $46 \%$ der von Tarifverträgen erfassten Beschäftigten. Für weitere 8,6 Mio. Beschäftigte traten Stufenerhöhungen in Kraft, die bereits 2011 oder früher vereinbart worden 
Ausgewählte Tarifforderungen und -abschlüsse in der Tarifrunde 2012

\begin{tabular}{|c|c|c|c|c|c|}
\hline Abschluss & Tarifbereich & Forderung & 2012 & 2013 & Laufzeit in Mon. \\
\hline 12.01. & Deutsche Post AG & $7,0 \%$ & $\begin{array}{l}400 € \text { Pauschale für } 3 \text { Monate } \\
4,0 \% \text { ab } 01.04 .12 \\
\text { bis } 31.03 .13\end{array}$ & & 15 \\
\hline 31.03. & $\begin{array}{l}\text { Öffentlicher Dienst } \\
\text { Bund und Gemeinden }\end{array}$ & $\begin{array}{l}6,5 \% \\
\text { mind. } 200 €\end{array}$ & $3,5 \%$ ab 01.03 .12 & $\begin{array}{l}1,4 \% \text { ab } 01.01 .13 \\
1,4 \% \text { ab } 01.08 .13 \\
\text { bis } 28.02 .14\end{array}$ & 24 \\
\hline 28.04 & Deutsche Telekom AG & $6,5 \%$ & $\begin{array}{l}3 \text { Nullmonate } \\
2,3 \% \text { ab } 01.05 .12\end{array}$ & $\begin{array}{l}2,1 \% \text { ab } 01.01 .13 \\
2,1 \% \text { ab } 01.08 .13 \\
\text { bis } 31.01 .14\end{array}$ & 24 \\
\hline 04.05. & Hotels und Gaststätten NRW & $6,0 \%$ & $\begin{array}{l}1 \text { Nullmonat } \\
\mathbf{3 , 1} \% \text { ab } 01.06 .12\end{array}$ & $\begin{array}{l}1,6 \% \text { ab } 01.02 .13 \\
1,6 \% \text { ab } 01.09 .13 \\
\text { bis } 30.04 .14\end{array}$ & 24 \\
\hline 08.05 & $\begin{array}{l}\text { Privates Verkehrsgewerbe } \\
\text { Baden-Württemberg } \\
\text { (ohne Südbaden) }\end{array}$ & $7,0 \%$ & $3,5 \%$ ab 01.04 .12 & $\begin{array}{l}2,5 \% \text { ab } 01.04 .13 \\
\text { bis } 31.03 .14\end{array}$ & 24 \\
\hline $\begin{array}{l}19.05 . / \\
22.05 .\end{array}$ & $\begin{array}{l}\text { Metall- und } \\
\text { Elektroindustrie }\end{array}$ & $6,5 \%$ & $\begin{array}{l}1 \text { Nullmonat } \\
4,3 \% \text { ab } 01.05 .12 \\
\text { bis } 30.04 .13\end{array}$ & & 13 \\
\hline 24.05. & Chemische Industrie & $6,0 \%$ & $\begin{array}{l}1 \text { Nullmonat } \\
4,5 \% \text { für } 18 \text { Monate } \\
\text { reg. unterschiedlich bis } 31.12 .13 \text {, } \\
\text { 31.01. bzw. } 28.02 .14\end{array}$ & & 19 \\
\hline 30.05 & $\begin{array}{l}\text { Kfz-Gewerbe } \\
\text { Baden-Württemberg }\end{array}$ & $6,5 \%$ & $\begin{array}{l}1 \text { Nullmonat } \\
4,0 \% \text { ab } 01.06 .12 \\
\text { bis } 31.05 .13\end{array}$ & & 13 \\
\hline 31.05. & Volkswagen AG & $6,5 \%$ & $\begin{array}{l}4,3 \% \text { ab } 01.06 .12 \\
\text { bis } 30.06 .13\end{array}$ & & 13 \\
\hline 06.06. & Bankgewerbe & $6,0 \%$ & $\begin{array}{l}350 € \text { Pauschale für } 4 \text { Monate } \\
2,9 \% \text { ab } 01.07 .12\end{array}$ & $\begin{array}{l}2,5 \% \text { ab } 01.07 .13 \\
\text { bis } 30.04 .14\end{array}$ & 26 \\
\hline 06.09 . & Papierverarbeitung & $6,5 \%$ & $\begin{array}{l}2 \text { Nullmonate } \\
\mathbf{3 , 1} \% \text { ab } 01.11 .12\end{array}$ & $\begin{array}{l}3,0 \% \text { ab } 01.11 .13 \\
\text { bis } 31.08 .14\end{array}$ & 24 \\
\hline 12.09. & Energiewirtschaft NRW (GWE) & $6,0 \%$ & $\begin{array}{l}2,95 \% \text { ab } 01.09 .12 \\
\text { bis } 31.10 .13\end{array}$ & & 14 \\
\hline 07.11. & Textil-Bekleidung West & $5,0 \%$ & 2 Nullmonate & $\begin{array}{l}240 € \text { Pauschale für } 4 \text { Monate } \\
\mathbf{3 , 0} \% \text { ab } 01.05 .13 \\
\mathbf{2 , 0} \% \text { ab } 01.06 .14 \text { bis } 31.10 .14\end{array}$ & 24 \\
\hline
\end{tabular}

Quelle:WSI-Tarifarchiv; Stand: 31.12. 2012.

sind. Bei rund 2,1 Mio. Beschäftigten liefen 2012 oder früher die Vergütungstarifverträge aus, aber es kam bis zum Jahresende (noch) nicht zu Neuabschlüssen bzw. laufende Tarifverträge sehen für 2012 keine Tarifanhebung vor.

\subsection{Abschlussrate}

Die tarifliche Abschlussrate beläuft sich gesamtwirtschaftlich im Durchschnitt auf 5,0 \% (2011: 4,8\%), in West- deutschland beträgt die Rate $4,9 \%$, in Ostdeutschland 5,6\%. Die Abschlussrate schließt alle, ggf. auch 2013 und später in Kraft tretenden tabellenwirksamen Erhöhungen ein. Nicht berücksichtigt werden Pauschalzahlungen und zusätzliche Einmalzahlungen, die sich nicht dauerhaft in den Tariftabellen niederschlagen. Die Spannweite der durchschnittlichen Gesamtabschlussraten reicht von 3,2 \% im Bereich Energie- und Wasserversorgung, Bergbau über 4,3\% im Grundstoff- und Produktionsgütergewerbe, 
Investitionsgütergewerbe, bis zu 6,3 \% im Bereich Gebietskörperschaften, Sozialversicherung.

Diese Gesamtabschlussraten sind nur von begrenzter Aussagekraft, weil sie sich immer auf die gesamte, je nach Tarifbereich sehr unterschiedlich lange Laufzeit der Tarifabkommen beziehen. Berücksichtigt man lediglich die im Jahr 2012 abgeschlossenen und auch in Kraft getretenen Tariferhöhungen, ergibt sich eine Abschlussrate von 3,9 \% (West: 3,9 \%, Ost: 3,8 \%). Differenziert man diese Größe nach Wirtschaftsbereichen, dann ergibt sich für 2012 eine Streuung zwischen 2,4 \% im Bereich Gartenbau, Land- und Forstwirtschaft und 4,2 \% im Grundstoff- und Produktionsgütergewerbe sowie im Investitionsgütergewerbe. Zu berücksichtigen ist, dass die Erhöhungen zu verschiedenen Zeitpunkten im Jahresverlauf wirksam wurden und damit auch die Auswirkungen auf die Tarifvergütungen des Jahres 2012 unterschiedlich waren.

Im Jahr 2012 spielten „Nullmonate” bei den Tarifabschlüssen eine deutlich geringere Rolle als noch im Vorjahr. Zwar gab es für rund 6,1 Mio. Beschäftigte (2011: 8,1 Mio.), das entspricht rund $68 \%$ der von Neuabschlüssen begünstigten Beschäftigten, Tarifabschlüsse mit verzögerter Anpassung der Lohn- und Gehaltserhöhungen. Aber die Zahl der Verzögerungsmonate blieb mit durchschnittlich 1,8 deutlich unter dem Vorjahr mit 3,2 Monaten.

Rund $76 \%$ von ihnen mussten einen Monat auf die reguläre Tariferhöhung warten, weitere $14 \%$ erhielten nach zwei bzw. drei Monaten ihre Tarifsteigerung, für die restlichen $10 \%$ dauerte es vier Monate und länger. Für 0,98 Mio., das sind $16 \%$ der davon betroffenen Beschäftigten, vereinbarten die Gewerkschaften als Ausgleich Pauschalzahlungen, die durchschnittlich $82 €$ (West: $82 €$, Ost: $85 €$ ) im Monat betrugen.

\subsection{Laufzeiten}

Der seit rund zehn Jahren zu beobachtende Trend zu fast zweijährigen Laufzeiten hat sich im vergangenen Jahr abgeschwächt. Die Laufzeit der Vergütungstarifverträge im Jahr 2012 beträgt durchschnittlich 18,0 (2011: 22,8 Monate). Für rund 4,6 Mio. Beschäftigte (52,1 \%) laufen die Abkommen zwischen elf und 15 Monate, für rund 0,9 Mio. (10,3\%) zwischen 16 und 23 Monate und für 3,3 Mio. (37,6 \%) zwei Jahre und länger. In den neuen Bundesländern laufen die neu abgeschlossenen Tarifverträge im Schnitt gut einen Monat länger als in den alten.

\subsection{Jahresbezogene Tarifsteigerung}

Bei der Berechnung der auf das Kalenderjahr bezogenen Steigerung der tariflichen Grundlöhne und -gehälter werden im Unterschied zur tariflichen Abschlussrate die Auswirkungen aus der unterschiedlichen Lage und Laufzeit der Tarifabkommen berücksichtigt. Auch werden ggf. im Berichtsjahr wirksam werdende Abschlüsse aus den Vorjahren sowie zusätzliche Einmalzahlungen und Pauschalzahlungen als Ausgleich für Abschlussverzögerungen mit einbezogen. Die jahresbezogene Tarifsteigerung setzt die durchschnittliche tarifliche Grundvergütung des gesamten Jahres 2012 zum Vorjahr in Bezug und erfasst insgesamt 17,5 Mio. Arbeitnehmerinnen und Arbeitnehmer.

Diese kalenderjährliche Steigerung der Tarifverdienste 2012 gegenüber 2011 beträgt für ganz Deutschland 2,7 \% (2011: 2,0 \%). Am höchsten fällt die jahresbezogene Tarifsteigerung mit 3,3 \% im Investitionsgütergewerbe aus, gefolgt vom Grundstoff- und Produktionsgütergewerbe mit 3,0 \%, dem Bereich Gartenbau, Land- und Forstwirtschaft mit 2,9\% und dem Nahrungs- und Genussmittelgewerbe mit 2,8 \%. Genau im Durchschnitt liegt mit 2,7 \% der Handel. Darunter liegen die Bereiche Private Dienstleistungen, Organisationen ohne Erwerbszweck sowie das Baugewerbe mit 2,5 \%, Verkehr und Nachrichtenübermittlung mit 2,3\%, Gebietskörperschaften, Sozialversicherung mit 2,2 \%, Verbrauchsgütergewerbe sowie Energie- und Wasserversorgung, Bergbau mit 2,1 \% und schließlich die Finanzdienstleistungen mit 2,0\%.

In Ostdeutschland liegt die kalenderjährliche Erhöhung mit 2,8 \% geringfügig höher als in Westdeutschland mit $2,7 \%$.

Leicht negativ beeinflusst wird die jahresbezogene Tarifsteigerung 2012 durch die länger laufenden Abschlüsse aus dem Jahr 2011. Die daraus resultierende Tarifanhebung für 2012 beläuft sich auf 2,5\%, die Neuabschlüsse des Jahres 2012 ergeben dagegen 2,9\%. Im Mittel ergeben sich dann die genannten $2,7 \%$.

In Tarifbereichen mit rund 622.000 Beschäftigten liefen Vergütungstarifverträge im Jahr 2012 aus, ohne dass bis zum Jahresende neue Abschlüsse getätigt wurden. Bezieht man diese Bereiche mit ein, ergibt sich eine jahresbezogene Tarifsteigerung von $2,6 \%$.

In Tarifbereichen mit weiteren 1,5 Mio. Beschäftigten sind die Tarifverträge bereits 2011 oder in den Jahren zuvor ohne nachfolgende Abschlüsse ausgelaufen. Berücksichtigt man auch diese Bereiche, dann sinkt die jahresbezogene Tarifsteigerung für alle von Tarifverträgen erfassten Beschäftigten für 2012 insgesamt auf 2,4\%.

Die durchschnittliche jahresbezogene Tarifsteigerung 2012 von 2,7 \% liegt über dem Anstieg der Lebenshaltungskosten von 2,0 \%. Real stiegen die tariflichen Grundvergütungen im gesamtwirtschaftlichen Durchschnitt um 0,7 \%.

\subsection{Entwicklung der Effektivverdienste}

Auch die Effektivverdienste zeigten im vergangenen Jahr eine Aufwärtstendenz: Die Summe der Bruttolöhne und -gehälter stieg 2012 um 3,7 \%. Je Beschäftigten ergibt sich auf Monatsbasis nominal ein Anstieg um 2,6\%, auf Stundenbasis sogar um 3,2 \%. Daraus ergibt sich, dass die Bruttoverdienste 2012 erneut real (preisbereinigt) gestiegen sind, und zwar um 0,6 \% auf Monatsbasis bzw. 1,2 \% auf Stundenbasis. Eine merkliche Lohndrift ist (auf Monatsbasis) nicht zu konstatieren. 
Der neutrale Verteilungsspielraum belief sich im vergangenen Jahr auf $1,8 \%$, darin sind der Anstieg der Verbraucherpreise $(+2,0 \%)$ und der Rückgang der Arbeitsproduktivität je Beschäftigten $(-0,3 \%)$ berücksichtigt. Berücksichtigt man dagegen die Stundenproduktivität $(+0,4 \%)$ ergibt sich ein Verteilungsspielraum von $2,4 \%$. Damit lag im vergangenen Jahr die tarifliche wie effektive Lohnentwicklung erkennbar über dem neutralen Verteilungsspielraum. Legt man dagegen als Maßstab die Zielinflationsrate der Europäischen Zentralbank (EZB) (2\%) und die Trendproduktivität (1,5\%) zugrunde, bleibt die Lohnentwicklung auch 2012 noch unter dieser Marke.

Betrachtet man die funktionelle Einkommensverteilung, so zeigt sich, dass im Jahr 2012 die Unternehmens- und Vermögenseinkommen um 1,4 \% zurückgingen, während die Arbeitnehmerentgelte um 3,6\% wuchsen.

\subsection{Lohnangleichung Ost/West}

Der Stand der tariflichen Lohnangleichung an das Westniveau kann an der Entwicklung der tariflichen Grundvergütung festgemacht werden. Für den Stichtag 31.12.2012 ergibt sich dabei folgendes Bild: Auf Basis von rund 50 Tarifbereichen/-branchen mit 1,64 Mio. erfassten Beschäftigten errechnet sich ein durchschnittliches Tarifniveau von 97,0 \%. Damit ergibt sich gesamtwirtschaftlich praktisch eine Steigerung gegenüber dem Vorjahr um 0,5 Prozentpunkte.

\subsection{Ausbildungsvergütungen}

Die Steigerung der tariflichen Ausbildungsvergütungen ist im vergangenen Jahr deutlich kräftiger ausgefallen als 2011. Nach Berechnungen des Bundesinstituts für Berufsbildung (BIBB) ergibt sich ein Anstieg von 4,3\% (West: 4,1\%, Ost: 5,0\%), der deutlich über der Steigerung der tariflichen Grundvergütungen liegt (BIBB 2013). Je nach Tarifbereich verbergen sich hinter diesen Durchschnittszahlen große Unterschiede: Gemessen an der Ausbildungsvergütung im dritten Ausbildungsjahr variierte die Steigerung in 26 ausgewählten Tarifbereichen zwischen 1,6 und 11,1 \%. In vier Tarifbereichen sind die Ausbildungsvergütungen im vergangenen Jahr regional oder bundesweit gar nicht angehoben worden.

\section{Regulierung der Leiharbeit}

Die Ausweitung der prekären Beschäftigung ist in den vergangenen Jahren zunehmend auch zum Gegenstand der gewerkschaftlichen Tarifpolitik geworden. Das betrifft zum einen den wachsenden Niedriglohnsektor, den die Gewerkschaften durch Mindestlohntarifverträge für einzelne Branchen und durch die gezielte Anhebung sehr niedriger Tariflöhne zu begrenzen versuchten. Zum anderen verstärkte der rasante Anstieg der Zahl der Leiharbeitsbeschäftigten
TABELLE 1

\section{Tarifsteigerung 2012}

Angaben in Prozent ${ }^{1}$

\begin{tabular}{|c|c|c|c|}
\hline Wirtschaftsbereich & Ost & West & Gesamt \\
\hline Gartenbau, Land- und Forstwirtschaft & 3,3 & 2,8 & 2,9 \\
\hline Energie- und Wasserversorgung, Bergbau & 3,3 & 1,9 & 2,1 \\
\hline Grundstoff- und Produktionsgütergewerbe & 3,1 & 3,0 & 3,0 \\
\hline Investitionsgütergewerbe & 3,4 & 3,3 & 3,3 \\
\hline Verbrauchsgütergewerbe & 2,3 & 2,1 & 2,1 \\
\hline Nahrungs- und Genussmittelgewerbe & 3,6 & 2,7 & 2,8 \\
\hline Baugewerbe & 2,7 & 2,5 & 2,5 \\
\hline Handel & 2,7 & 2,7 & 2,7 \\
\hline Verkehr und Nachrichtenübermittlung & 2,7 & 2,3 & 2,3 \\
\hline Kreditinstitute, Versicherungsgewerbe & 2,0 & 2,0 & 2,0 \\
\hline Priv. Dienstleistungen, Organ. o. Erwerbszweck & 2,9 & 2,4 & 2,5 \\
\hline Gebietskörperschaften, Sozialversicherung & 2,2 & 2,2 & 2,2 \\
\hline Gesamte Wirtschaft & 2,8 & 2,7 & 2,7 \\
\hline
\end{tabular}

1 Jahresbezogene Erhöhung der tariflichen Grundvergütung 2012 gegenüber 2011. Quelle:WSI-Tarifarchiv; Stand: 31.12.2012.

bis zur Finanzkrise und auch danach die Befürchtung, dass in vielen Betrieben dauerhaft eine zweite, deutlich niedrigere Lohnlinie etabliert wird und dadurch die bestehenden Tarifniveaus ausgehöhlt werden. Namentlich die IG Metall hatte bereits vor einigen Jahren eine Kampagne gestartet, die auf betrieblicher, tarifpolitischer und gesellschaftspolitischer Ebene ihre Forderungen verankern sollte. Unter der Überschrift „Leiharbeit fair gestalten“ zielte die Initiative „Gleiche Arbeit - Gleiches Geld“ der IG Metall seit ihrem Start im April 2008 im Kern auf die Durchsetzung des Equal Pay-Grundsatzes für die Leiharbeit. ${ }^{\bullet}$ In der Tarifrunde 2012 stellte die IG Metall dann konkrete Forderungen zur Ausweitung der Mitbestimmung des Betriebsrates beim Einsatz von Leiharbeit und zur Angleichung der Bezahlung von Leiharbeitskräften und Stammbelegschaft auf Basis von Branchenzuschlägen und Einsatzzulagen.

In einem neuen Tarifvertrag Leih-/Zeitarbeit wurden dann für die Metall- und Elektroindustrie folgende Regelungen getroffen:

- Der Einsatz von Leiharbeitsbeschäftigten darf im Entleihbetrieb nicht zur Beeinträchtigung der Entgelt-/Arbeitsbedingungen führen und Arbeitsplätze gefährden.

Ein gesetzlicher Mindestlohn sollte zudem Leiharbeit zu Armutslöhnen verhindern, das Synchronisationsverbot sollte wieder eingeführt werden, um das „Heuern und Feuern“ zu unterbinden, und eine Höchstverleihzeit sollte verhindern, dass Leiharbeit als Instrument zum Abbau von Stamm-Arbeitsplätzen genutzt wird (http://www.gleichearbeitgleichesgeld.de/initiative/gruendungserklaerung/). 
- Ein Einsatz ist u. a. zulässig bei zeitlicher Befristung, Vorlage eines Sachgrundes oder Abarbeitung von Auftragsspitzen.

- Der Einsatz von Leiharbeitnehmerinnen und Leiharbeitnehmern bedarf grundsätzlich der Zustimmung des Betriebsrats im Entleihbetrieb.

- Ist der Einsatz von Leiharbeitsbeschäftigten für länger als drei Monate vorgesehen, muss auf Verlangen des Betriebsrats eine innerbetriebliche Stellenausschreibung erfolgen.

- Nach 18/24 Monaten Einsatzdauer: Der Entleiher prüft Möglichkeit des Angebots/grundsätzlich Angebot eines unbefristeten Arbeitsvertrags.

- Es erfolgt eine Addition der Einsatzzeiten im selben Betrieb bei Unterbrechungszeiten unter drei Monaten.

- Es besteht die Möglichkeit zum Abschluss einer freiwilligen Betriebsvereinbarung, u. a. mit Regelungen zu Einsatzzweck, -bereichen und Volumen von Leiharbeit, Höhe der Vergütung, Einsatzhöchstdauer, Übernahme in ein unbefristetes Arbeitsverhältnis.

- Durch eine Betriebsvereinbarung können folgende Möglichkeiten geschaffen werden: Erhöhung der Quote (max. 12 Prozentpunkte) der Arbeitnehmerinnen und Arbeitnehmer mit Arbeitszeit-Verlängerung auf bis zu 40 Std./Woche; im gleichen Volumen Angebot von Arbeitszeit-Reduzierung auf bis zu 30 Std./Woche (verkürzte Vollzeit mit Rückkehrrecht); bei Übernahme von Leiharbeitsbeschäftigten Auszahlung von Guthaben aus den Arbeitszeit-Konten.

- Der Betriebsrat hat ein Recht auf Information über Umfang und Einsatzbereiche von Leiharbeit sowie auf Einsichtnahme in die Verträge zwischen Arbeitgeber und Verleiher. - Der Entleiher ist dem Verleiher verpflichtet, ihn über betriebliche Regelungen zugunsten der Leiharbeitnehmerinnen und Leiharbeitnehmer zu informieren.

In parallelen Tarifverhandlungen mit den beiden Zeitarbeitsverbänden BAP (früher BZA) und iGZ ging es um die Festlegung von Branchenzuschlägen. Hier konnte die IG Metall folgende Regelungen vereinbaren:

- Nach der sechsten Woche bzw. dem 3./5./7./9. Monat ununterbrochener Einsatzdauer in einem Kundenbetrieb wird ein Zuschlag von 15/20/30/45/50 \% auf Basis der Vergütungen der DGB-BZA/iGZ-Tarifverträge gezahlt.

- Bei Unterbrechungszeiten unter drei Monaten erfolgt eine Addition der Einsatzzeiten im selben Betrieb.

- Der Zuschlag wird auf die Differenz der Vergütung von Leiharbeitsbeschäftigten und vergleichbaren Beschäftigten des Entleihbetriebs ohne Berücksichtigung des Äquivalents einer durchschnittlichen Leistungszulage (10 \%) begrenzt. - Leiharbeitnehmerinnen und Leiharbeitnehmer erhalten den Branchenzuschlag auch, wenn sie in nicht tarifgebundenen Metall- und Elektrounternehmen arbeiten.

- Wegfall der einsatzbezogenen Zulage gemäß $₫ 4$ ETV bzw. $\$ 5$ ERTV zwischen DGB und BZA bzw. iGZ.

- Anspruch auf Leistungen entsprechend den betrieblichen Vereinbarungen im Entleihbetrieb.
- Verfahrensregelung zur Anpassung des Branchenzuschlags an Tariferhöhungen.

Der Tarifvertrag trat zum 1. November 2012 in Kraft und hat eine Laufzeit bis Ende 2017. Die IG Metall bezifferte den Zuschlag für einen Leiharbeitsbeschäftigten in der untersten Entgeltgruppe je nach Einsatzdauer zwischen $186,33 €$ und 621,09€ pro Monat und wertete den Vertrag als einen „wichtigen Schritt hin zur fairen Bezahlung“von Leiharbeitsbeschäftigten.

Die IG Metall stellte heraus, es sei erstmals gelungen, die Einsatzbedingungen von Leiharbeitsbeschäftigten in einem Tarifvertrag zu beschreiben und den Betriebsräten mehr Mitbestimmungsrechte einzuräumen. Das stärke die Position der Betriebsräte „gewaltig“, so Bezirksleiter Jörg Hofmann (siehe dazu König/Detje 2012). Die Metallarbeitgeber betonten demgegenüber, dass die Betriebe in den ersten zwei Jahren nach wie vor selbst entscheiden könnten, ob und wie sie Leiharbeitsbeschäftigte einsetzen wollten. Aus ihrer Sicht sind vor allem die Bestimmungen zur Arbeitszeitflexibilisierung als Ausgleich für eine eventuelle Beschränkung der Leiharbeit von zentraler Bedeutung. Die IG Metall wies darauf hin, dass unabhängig von diesen tarifpolitischen Fortschritten der Gesetzgeber in Sachen ReRegulierung der Leiharbeit nach wie vor gefordert bleibt (vgl. auch Burmeister 2012).

Der Abschluss stieß bei den anderen Gewerkschaften auf zum Teil deutliche Kritik (Völpel 2012; Wiedemuth 2012). Insbesondere wurde bemängelt, dass damit das Ziel von „Equal Pay“ nicht verwirklicht und die Politik aus der Regelungsverantwortung entlassen werde. Auch sei das Modell nicht auf alle Branchen gleichermaßen übertragbar. Dennoch vereinbarten einige Gewerkschaften in anderen Branchen weitere Abschlüsse, die sich in der Grundstruktur am Metallabschluss orientierten. Die Zuschläge fielen in Abhängigkeit von den Vergütungsniveaus in den Branchen unterschiedlich aus (Übersicht 2).

\section{Förderung der Ausbildung}

Die Verbesserung der Situation im Bereich der beruflichen Ausbildung ist seit Langem ein Thema der Tarifpolitik (Bispinck et al. 2002). Dabei geht es zum einen um die Sicherstellung eines ausreichenden quantitativen Ausbildungsplatzangebots und zum anderen um die Perspektive der gesicherten Übernahme der Auszubildenden nach (erfolgreichem) Abschluss ihrer Ausbildung. In jüngster Zeit mehren sich auch die Aktivitäten zur Förderung von Jugendlichen mit unzureichenden Ausbildungsvoraussetzungen.

Was die Übernahme nach der Ausbildung betrifft, gibt es in zahlreichen Tarifverträgen einschlägige Regelungen. Überwiegend handelt es sich um befristete Übernahmere- 
gelungen mit einem Zeitraum zwischen sechs und 24 Monaten, mehrheitlich mit zwölf Monaten. Vorschriften für eine unbefristete Übernahme wurden bislang meist in Firmentarifverträgen vereinbart, erstmals kam es 2011 in der Stahlindustrie zu einer branchenweiten Regelung über eine unbefristete Übernahme. In einigen Branchen existieren dagegen keinerlei tarifliche Übernahmeregelungen. Zu diesem Ergebnis kommt eine Analyse des WSI-Tarifarchivs von Tarifverträgen in 50 Branchen und 15 Firmentarifverträgen (Bispinck/WSI-Tarifarchiv 2012).

In den meisten Fällen ist die Übernahme ,grundsätzlich“ vorgesehen bzw. als Sollvorschrift ausgestaltet. Die Betriebe können z. B. beim Vorliegen verhaltens- oder personenbedingter Gründe oder wegen akuter Beschäftigungsprobleme von der Übernahme Abstand nehmen. Eine weitere Variante stellt die Übernahme in ein Teilzeitarbeitsverhältnis dar. Diese teilzeitige Übernahme ist manchmal als zweitbeste Lösung vorgesehen, wenn eine vollzeitige Beschäftigung nicht möglich erscheint. Auch ist die Möglichkeit einer stufenweisen Aufstockung auf Vollzeit vorgesehen. Diese Regelungen zeigen, dass die Tarifparteien das Instrumentarium der Tarifpolitik durchaus nutzen, aber sie schöpfen das vorhandene Potenzial keinesfalls aus.

Im Tarifjahr 2012 spielte dieses Thema in verschiedenen Branchen eine Rolle, insbesondere in der Metall- und Elektroindustrie: Bereits im Januar 2009 hatte die IG MetallJugend eine Kampagne „Operation Übernahme“ auf den Weg gebracht, die vor allem auf die unbefristete Übernahme der Ausgebildeten durch den ausbildenden Betrieb zielte (www.operationuebernahme.de). In der Tarifrunde 2012 sollte die unbefristete Übernahme tarifvertraglich durchgesetzt werden. Folgende Regelungen wurden dann hierzu vereinbart:

- Die Auszubildenden „sollen“ in der Regel nach bestandener Abschlussprüfung unbefristet in ein Arbeitsverhältnis übernommen werden.

- Nach Möglichkeit soll die Anzahl an Ausbildungsplätzen gesteigert, mindestens aber beibehalten werden.

- Möglichkeit einer freiwilligen Betriebsvereinbarung zur Festschreibung des Ausbildungsbedarfs und Festlegung der Anzahl der unbefristet zu übernehmenden Ausgebildeten; über Bedarf Ausgebildete besitzen keinen Anspruch auf Übernahme.

- Wird keine Betriebsvereinbarung abgeschlossen, beraten Betriebsrat und Arbeitgeber jeweils spätestens sechs Monate vor Ende der Ausbildung über die Anzahl der unbefristet zu übernehmenden Ausgebildeten. Über Bedarf Ausgebildete besitzen einen Anspruch auf Übernahme für mindestens zwölf Monate.

- Eine Abweichung von der Übernahmeverpflichtung ist möglich, u. a. bei Vorliegen akuter Beschäftigungsprobleme.

Zum Metall-Abschluss gehört im Tarifgebiet Baden-Württemberg auch noch eine Sozialpartner-Vereinbarung „Vom Einstieg zum Aufstieg“, die einen Beitrag zur Stärkung des

\section{ÜBERSICHT 2}

\section{Tarifliche Branchenzuschläge bei Arbeitnehmerüberlassung}

Zuschläge in Prozent nach Einsatzdauer

\begin{tabular}{|c|c|c|c|c|c|c|}
\hline & Gruppe & $\begin{array}{c}\text { ab } \\
\text { 7. Woche }\end{array}$ & $\begin{array}{c}\text { ab } \\
\text { 4. Monat }\end{array}$ & $\begin{array}{c}\text { ab } \\
\text { 6. Monat }\end{array}$ & $\begin{array}{c}a b \\
\text { 8. Monat }\end{array}$ & $\begin{array}{c}\text { ab } \\
\text { 10. Monat }\end{array}$ \\
\hline \multirow[t]{3}{*}{ Chemie } & $\mathrm{E} 1-\mathrm{E} 2$ & 15 & 20 & 30 & 45 & 50 \\
\hline & E3 - E5 & 10 & 14 & 21 & 31 & 35 \\
\hline & E6 - E9 & 0 & 0 & 0 & 0 & 0 \\
\hline \multirow[t]{4}{*}{ Eisenbahn } & $\mathrm{E} 1-\mathrm{E} 2$ & 4 & 6 & 8 & 12 & 14 \\
\hline & E3 & 3 & 4 & 6 & 9 & 10 \\
\hline & E4 - E5 & 4 & 6 & 8 & 12 & 14 \\
\hline & E6 - E9 & 0 & 0 & 0 & 0 & 0 \\
\hline Holz und Kunststoff & E1 - E9 & 7 & 10 & 15 & 22 & 31 \\
\hline \multirow[t]{4}{*}{ Kautschuk } & $\mathrm{E} 1-\mathrm{E} 2$ & 4 & 7 & 10 & 13 & 16 \\
\hline & E3 & 3 & 4 & 6 & 9 & 10 \\
\hline & $\mathrm{E} 4-\mathrm{E} 6$ & 4 & 7 & 10 & 13 & 16 \\
\hline & E7 - E9 & 0 & 0 & 0 & 0 & 0 \\
\hline \multirow[t]{4}{*}{ Kunststoffverarbeitung } & $\mathrm{E} 1-\mathrm{E} 2$ & 7 & 10 & 15 & 22 & 25 \\
\hline & $\mathrm{E} 3-\mathrm{E} 4$ & 4 & 6 & 9 & 13 & 15 \\
\hline & E5 & 3 & 4 & 6 & 9 & 10 \\
\hline & E6 - E9 & 0 & 0 & 0 & 0 & 0 \\
\hline Metall und Elektro & E1 - E9 & 15 & 20 & 30 & 45 & 50 \\
\hline Papierverarbeitung & $\mathrm{E} 1-\mathrm{E} 9$ & $\begin{array}{c}\text { (ab 5. Woche) } \\
4\end{array}$ & 8 & 12 & 16 & 20 \\
\hline Tapetenindustrie & $\mathrm{E} 1-\mathrm{E} 9$ & $\begin{array}{c}\text { (ab 5. Woche) } \\
7\end{array}$ & 11 & 15 & 19 & 23 \\
\hline Textil-Bekleidung & E1 - E9 & 5 & 10 & 15 & 20 & 25 \\
\hline
\end{tabular}

Quelle:WSI-Tarifarchiv - Stand: Dezember 2012.

Fachkräftepotenzials leisten soll. Jugendliche mit Lerndefiziten sollen gefördert, Ausbildungskapazitäten erhalten, gesteigert und die berufliche Entwicklung durch Weiterbildung soll vorangetrieben werden. Im Rahmen dieser Vereinbarung wurde auch erstmals ein Tarifvertrag „Förderjahr“ abgeschlossen, der konkrete Anforderungen und Maßnahmen für Abschluss und Durchführung eines Fördervertrages mit förderbedürftigen Jugendlichen beschreibt. In anderen Regionen gab es ebenfalls Vereinbarungen zu dieser Thematik (vgl. Hay et al. 2013 zu den bisherigen Erfahrungen in der Metallindustrie NRW).

Auch in der chemischen Industrie wurde das Thema in der Tarifrunde behandelt: Die Tarifvertragsparteien verständigten sich darauf, die einzelnen Bausteine für eine Karriere in der chemischen Industrie vor dem Hintergrund der demografischen Entwicklung weiterzuentwickeln und legten dazu einen konkreten Handlungskatalog „Attraktive Perspektiven für Berufseinsteiger in der Chemie-Branche“ vor.

Die Forderung nach unbefristeter Übernahme wurde 2012 auch in einigen anderen Branchen und Tarifbereichen aufgestellt (Öffentlicher Dienst [Bund, Gemeinden], KfzGewerbe, Banken, Versicherungsgewerbe u. a.). Eine verbindliche Festschreibung gelang in Firmentarifverträgen der 
Energiewirtschaft, ansonsten blieb es bei Empfehlungen bzw. bei einer zunächst befristeten Regelung.

\section{Tarifliche Mindestlöhne}

Anfang 2013 bestanden in zwölf Branchen tarifliche Mindestlöhne auf der Basis des Arbeitnehmerentsendegesetzes (AEntG) bzw. dem Arbeitnehmerüberlassungsgesetz (AÜG). Im Laufe des Jahres 2012 traten in neun Branchen Erhöhungen der Mindestlöhne zwischen 1,0 und 14,9 \% in Kraft (Übersicht 3). In drei Branchen waren die bereits vereinbarten Mindestlöhne zum Zeitpunkt des Redaktions- schlusses noch nicht in Kraft getreten (Gerüstbauerhandwerk) bzw. die Branchen noch nicht im Geltungsbereich des AEntG enthalten (Steinmetz- und Steinbildhauerhandwerk, Forstliche Dienstleister).

\section{Ausblick}

In einer Reihe von Tarifbereichen wurden bereits Tariferhöhungen für das Jahr 2013 und 2014 vereinbart. Die Abschlussraten bewegen sich zwischen 2,1 und 3,0\%. Auch in der Tarifrunde 2013 stehen Forderungen nach deutlichen Steigerungen der Tarifentgelte im Mittelpunkt. Das Spek-

ÜBERSICHT 3

Tarifliche Mindestlöhne nach AEntG

nur Branchen mit Steigerungen 2012

\begin{tabular}{|c|c|c|c|c|c|}
\hline Branche & Beschäftigten-/Entgeltgruppe & $\begin{array}{c}\text { Jan. } 2012 \\
\text { in } €\end{array}$ & $\begin{array}{l}\text { Jan. } 2013 \\
\text { in } €\end{array}$ & $\begin{array}{l}\text { Diff. } \\
\text { in } €\end{array}$ & $\begin{array}{l}\text { Diff. } \\
\text { in \% }\end{array}$ \\
\hline Abfallwirtschaft & Mindestlohn & 8,33 & 8,68 & 0,35 & 4,2 \\
\hline \multicolumn{6}{|l|}{ Bauhauptgewerbe } \\
\hline \multirow[t]{2}{*}{ West } & Werker (inkl. Berlin) & 11,05 & 11,05 & & \\
\hline & Fachwerker & 13,40 & 13,70 & 0,30 & 2,2 \\
\hline Berlin & Fachwerker & 13,25 & 13,55 & 0,30 & 2,3 \\
\hline Ost & Werker & 10,00 & 10,25 & 0,25 & 2,5 \\
\hline Dachdeckerhandwerk & Mindestlohn & 11,00 & 11,20 & 0,20 & 1,8 \\
\hline \multicolumn{6}{|l|}{ Elektrohandwerk (Montage) } \\
\hline West & Mindestentgelt & 9,80 & 9,90 & 0,10 & 1,0 \\
\hline Ost inkl. Berlin & Mindestentgelt & 8,65 & 8,85 & 0,20 & 2,3 \\
\hline \multicolumn{6}{|l|}{ Gebäudereinigerhandwerk } \\
\hline \multirow[t]{2}{*}{ West inkl. Berlin } & Innen- und Unterhaltsreinigung & 8,82 & 9,00 & 0,18 & 2,0 \\
\hline & Glas- und Fassadenreinigung & 11,33 & 11,33 & & \\
\hline \multirow[t]{2}{*}{ Ost } & Innen- und Unterhaltsreinigung & 7,33 & 7,56 & 0,23 & 3,1 \\
\hline & Glas- und Fassadenreinigung & 8,88 & 9,00 & 0,12 & 1,4 \\
\hline Gerüstbauerhandwerk & Mindestlohn & 9,50 & 10,00 & 0,50 & 5,3 \\
\hline \multicolumn{6}{|l|}{ Maler- und Lackiererhandwerk } \\
\hline \multirow[t]{2}{*}{ West } & ungelernter AN & 9,75 & 9,75 & 0,00 & \\
\hline & Geselle & 11,75 & 12,00 & 0,25 & 2,1 \\
\hline Ost & ungelernter AN & 9,75 & 9,75 & 0,00 & \\
\hline Wach- und Sicherheitsgewerbe & Mindestlohn & & & & \\
\hline West (regional unterschiedlich) & & $6,53-8,60$ & $7,50-8,90$ & $0,24-0,97$ & $3,3-14,9$ \\
\hline Ost inkl. Berlin & & 6,53 & 7,50 & 0,97 & 14,9 \\
\hline \multicolumn{6}{|c|}{ Wäschereidienstleistungen im Objektkundegeschäft } \\
\hline West & Mindestlohn & 7,80 & 8,00 & 0,20 & 2,6 \\
\hline Ost inkl. Berlin & Mindestlohn & 6,75 & 7,00 & 0,25 & 3,7 \\
\hline Arbeitnehmerüberlassung & Lohnuntergrenze & & & & \\
\hline West & & 7,89 & 8,19 & 0,30 & 3,8 \\
\hline Ost inkl. Berlin & & 7,01 & 7,50 & 0,49 & 7,0 \\
\hline
\end{tabular}


trum der Tarifforderungen reicht von 5,0-6,6\%. In der öffentlichen Diskussion mehren sich die Stimmen von Ökonomen, dass überdurchschnittliche Lohnsteigerungen auch mit Blick auf die Eurokrise erforderlich seien. So sprach sich der Präsident des Deutschen Instituts für Wirtschaftsforschung (DIW) Gert G. Wagner für Lohnsteigerungen von „im Durchschnitt $4 \%$ oder mehr" aus. Das Mitglied des Sachverständigenrates Peter Bofinger plädierte für „5 \% Plus über alle Branchen“ hinweg. Darin sei ein zweiprozentiger „Zuschlag zur Euro-Rettung“ enthalten. Kräftige Lohnsteigerungen stimulierten die Binnennachfrage und schafften indirekt auch Spielräume für die Lohnentwicklung in den Krisenländern. Dies traf erwartungsgemäß auf Widerspruch seitens der Arbeitgeberverbände. BDAPräsident Dieter Hundt plädierte für eine Fortsetzung des Kurses einer „verantwortungsvollen“ Tarifpolitik, die auf Flexibilität und Differenzierung setzt (Hundt 2013).

Nach dem Kündigungsterminkalender werden im ersten Halbjahr 2013 folgende größere Tarifbereiche das Tarifgeschehen bestimmen: der öffentliche Dienst (Länder), wo die Verträge bereits zum Jahresende ausgelaufen sind. Im Februar folgt die Eisen- und Stahlindustrie, Ende März das Bauhauptgewerbe, das Versicherungsgewerbe und erste Bereiche des Einzel-, Groß- und Außenhandels. Ende April stehen die Verträge in der Metall- und Elektroindustrie auf dem Tarifkalender.

\section{LITERATUR}

Bispinck, R./Dorsch-Schweizer, M. /Kirsch, J. (2002): Tarifliche Ausbildungsförderung begrenzt erfolgreich - eine empirische Wirkungsanalyse, in: WSI-Mitteilungen 55 (2), S. 213-219, http://www.boeckler.de/pdf/wsimit_2002_04_ bispinck.pdf

Bispinck, R./WSI-Tarifarchiv (2009): Tarifpolitischer Jahresbericht 2008: Tarifpolitik in der Finanzmarktkrise, Düsseldorf

Bispinck, R./WSI Tarifarchiv (2012): Förderung der Ausbildung durch Tarifvertrag im Jahr 2011, Tarifliche Regelungen zur Schaffung von Ausbildungsplätzen und zur Übernahme von Ausgebildeten, Elemente qualitativer Tarifpolitik (74), Düsseldorf

Bundesinstitut für Berufsbildung (BIBB) (2013): Tarifliche Ausbildungsvergütungen 2012: Kräftiger Anstieg in West und Ost, Pressemeldung vom 9.1.
Bundesagentur für Arbeit (BA) (2013): Der Arbeits- und Ausbildungsmarkt in Deutschland, Monatsbericht Dezember und Jahr 2012

Burmeister, K. (2012): Leiharbeit - Tarif-Erfolg braucht Unterstützung durch linke Politik, in: Zeitschrift für Sozialistische Politik und Wirtschaft (SPW) 3/2012, S. 4

Hay, Th./Nettelstroth, W./Hüsson, N. (2013): Tarifpolitische Initiativen zum Aus bildungseinstieg und zur Übernahme von Ausgebildeten, in: WSI-Mitteilungen 66 (1), S. 57-60, http://www.boeckler.de/wsimit_2013_01_nettelstroth.pdf Hundt, D. (2013): Tarifpolitik - Ausblick 2013. Kurs einer verantwortungsvollen Tarifpolitik fortsetzen, Statement auf der Bundespressekonferenz am 22.1. Institut für Makroökonomie und Konjunkturforschung, Arbeitskreis Konjunktur (IMK) (2011): Im Bann der Austeritätspolitik - Prognose der wirtschaftlichen Entwicklung 2012, IMK Report (69), Dezember

König, O./Detje, R. (2012): Fuß in derTür. Tarifabschluss Metall- und Elektroindustrie 2012: Junge Metaller gewinnen, in: Sozialismus 39 (365), S. 48-50 Roach, M. (2012): Tarifliche Regelungen zum Gesundheitsschutz im Bankgewerbe, Präsentation auf der Tarifpolitischen Tagung des WSI am 27. 09., http:// www.boeckler.de/pdf/v_2012_09_27_roach.pdf

Spiegel Online (2012): Zahltag bei den Dax-Konzernen: Der rasende Anstieg der Chefgehälter, 19.03., http://www.spiegel.de/wirtschaft/unternehmen/entwicklung-der-gehaelter-von-dax-vorstandschefs-a-821327.html (letzter Zugriff: 21.01.2013)

Völpel, E. (2012): Leiharbeiter in der Industrie - Ein bisschen Zuschlag, taz vom 20.8 .

Wiedemuth, J. (2012): Branchenzuschläge in der Leiharbeit - eine Alternative zu Equal-Pay?, in: ver.di-tarif.letter 3/2012, S. 1-6

\section{AUTOREN}

REINHARD BISPINCK, Dr., ist Wissenschaftler im Wirtschafts- und Sozialwissenschaftlichen Institut (WSI) und Leiter des WSI-Tarifarchivs in der HansBöckler-Stiftung.

reinhard-bispinck@boeckler.de

GÖTZ BAUER, ULRICH SCHMIDT, MONIKA SCHWACKE-PILGER, KATHRIN SONNEN, ANDREA TAUBE und MONIKA WIEBEL sind Sachbearbeiterinnen und Sachbearbeiter im WSI-Tarifarchiv. 